\title{
Water Hydrogen Bonds Study by Opto-Magnetic Fingerprint Technique
}

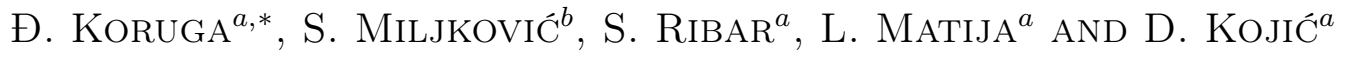 \\ ${ }^{a}$ NanoLab, Faculty of Mechanical Engineering, University of Belgrade \\ Kraljice Marije 16, 11120 Belgrade, Serbia \\ ${ }^{b}$ Galenika Institute, Batajnički drum bb, 11080 Belgrade, Serbia
}

\begin{abstract}
Hydrogen bond has dual property, classical (electrostatic interaction based on Coulomb's law) and quantum (wave function based on Schrödinger equation). Since Planck's constant is one of the main criteria for decision which process is quantum, or how much is close to be quantum, we use electrical and magnetic forces of valence electrons, as point of departure, to develop method for opto-magnetic fingerprint of matter. During the study of different type of matter we observed phenomena from spectral convolution data of digital images which characterize matter from both covalent and non-covalent bonding. Since water is matter that is most abundant with hydrogen bonds, we present results of $18.2 \mathrm{M} \Omega$ water investigation on different temperature and under influence of constant and variable magnetic fields by opto-magnetic method. Bearing in mind that Linus Pauling, in his book Nature of the Chemical Bond (Cornel University Press, 1939), for the first time presented the systematic concept of the hydrogen bond to the molecular world and its machinery, this paper is written in honor to him and 70th anniversary of one of the most important scientific paradigm.
\end{abstract}

PACS numbers: 33.20.Kf, 33.57.+c, 42.25.Ja, 42.30.-d, 42.50.Ex

\section{Introduction}

Hydrogen bond and its nature have been occupying the attention of scientific community since 1902, when German scientist Werner used words nebenvalenz and innere komplexsalzbildung to describe intra- and intermolecular bonds which are not covalent [1]. However, hydrogen bond became common term when Pauling gave systematic concept of the hydrogen bond in the chemistry [2]. In spite that he proposed that hydrogen bond in water is not only classical electrical attraction between a positively charged hydrogen atom and a negatively charged oxygen atom, but it also should be affected by the sigma bonds, no one took his proposal seriously until 1999, when it was experimentally shown that hydrogen bonds possess covalence and have both classical and quantum properties [3].

On the basis of data obtained from neutron diffraction experiments it was made clear that distance between center of hydrogen and oxygen atoms in a covalent bond $d(\mathrm{O}-\mathrm{H})$ of different structures is between $95 \mathrm{pm}$ and $120 \mathrm{pm}$, while distance of center of hydrogen and oxygen atoms in non-covalent bond $d(\mathrm{O} \ldots \mathrm{H})$ is between $120 \mathrm{pm}$ and $200 \mathrm{pm}$. However, for each type of matter product value $d(\mathrm{O}-\mathrm{H}) \times d(\mathrm{O} \ldots \mathrm{H})$ is about $162 \mathrm{pm}[4,5]$. Systematic investigation and quantitative analysis of bond lengths of $\mathrm{O}-\mathrm{H}$... O showed that bond-valence parameters of hydrogen bonds follow Golden ratio rule, whose value is around $1.62[6-8]$. Since the Golden mean naturally means harmonization, those values (162 pm and

\footnotetext{
* corresponding author; e-mail: dkoruga@mas.bg.ac.rs
}

1.62) indicate that value of natural measure should be two order of magnitude bigger than $162 \mathrm{pm}$. From hydrogen bond point of view natural measure (unit) of molecular world is $1.61803 \times 10^{-10} \mathrm{~m}$, or $1.61803 \AA$.

Bearing in mind that water is matter that is most abundant with hydrogen bonds, which may be organized in molecular networks, this indicates that water via hydrogen bonds (with classical and quantum properties), may play a role in molecular and biomolecular recognition. From this point of view, two primary goals in today pharmacy are: (1) understanding mechanism of molecular recognition in water solution, and (2) water structure for drug design. At present time, some pharmacologists are becoming aware of importance of water structure for drug design, because modeling ligand-receptor interaction has to include specific geometry, which relates to water structure [9]. It is well known that hydrogen bonds are a link between two nucleotide chains in DNA and support existence of secondary, ternary and quaternary structure of proteins. Since hydrogen bonds play important role in water, biomolecular structures, hydrated crystals and nanostructures we started to do research to characterize water and its hydrogen bonds by opto-magnetic method. By this method, based on light-water interaction, it is possible to collect data of both classical and quantum actions of water molecules and interactions between them.

\section{Method and material}

This method is based on light-matter interaction and ratio of electrical and magnetic forces of covalent bonds and intermolecular bonds of matter. DNA research 
indicates that both classical and quantum mechanical approach give same phenomenological results for those structures [10]. Reason for similar result is simple one: for stationary quantum state Hamiltonian is a sum of kinetic $(T)$ and potential $(V)$ energy, while Lagrangian is a difference between them when system is in equilibrium with external forces. Two similar pictures, one classical and another quantum, of the same object with similar results from the energy point of view do exist. Our goal is to find out how hydrogen bonds participate in water to be more or less classical or quantum entity. To give answer to this question we use Planck's constant $(h)$ as the first criteria to estimate whether an object is classical or quantum. Since Planck's constant by nature is action then product of force $(F)$, distance $(d)$ and time $(t)$ of action has to have value $h\left(6.626 \times 10^{-34} \mathrm{Js}\right)$, or close to if system is quantum one. However, what will be value for coupling quantum-classical system, and when classical one becomes dominant, it is unknown, and needs answer.

Planck's constant is link between energy $(E)$ and electromagnetic wave oscillation $(\nu)$, as $E=h \nu$. Comparison of the electrical and magnetic interaction between two electron charges in neighbouring atoms in relative motion within a matter may give a solution. We know that it is not easy to calculate the magnetic interaction between two charged particles in motion relative to an observer $\mathrm{O}$ in a form similar to the electric interaction given by Coulomb's law. However, we may compare an order of the magnitude of the magnetic interaction compared to electrical interaction. Considering two charges $q$ and $q^{\prime}$ of neighbouring atoms moving with velocities $v$ and $v^{\prime}$ relative to an observer, we may simplify the formulas because we want only order of magnitude. Thus, we can say that the electrical force produced by $q^{\prime}$ on $q$ as measured by $\mathrm{O}$ is $q E$. The magnetic field produced by $q^{\prime}$, if we use equation $B=1 / c^{2}(v \times E)$, is of the order of magnitude of $v^{\prime} E / c^{2}$ and hence the magnetic force on $q$ is of the order of $q v B=\left(v v^{\prime} / c^{2}\right) q E$. Since, $q E$ is the electrical force on $q$, then required ratio magnetic force/electrical force is $\left(F_{\mathrm{M}} / F_{\mathrm{E}}\right) \approx v v^{\prime} / c^{2}$. If the velocities of the charges are small compared to the velocity of light $c$, the magnetic force is negligible compared to the electrical force and in many cases can be ignored. The orbital velocity of valence electrons in atoms is about $10^{6} \mathrm{~m} / \mathrm{s}$, which gives $F_{\mathrm{M}} / F_{\mathrm{E}} \approx 10^{-4}$. This means that existence of semi-classical/quantum could be in the interval $6.626 \times 10^{-34}<h^{*}<6.626 \times 10^{-30}$. In this action area, from the energy point of view, both classical and quantum phenomena simultaneously exists. So this action area is perfect one for hydrogen bond investigation: if $h^{*} \leq 6.626 \times 10^{-30}$ Js then it is classical, while if $h^{*} \geq 6.626 \times 10^{-34} \mathrm{Js}$, it is quantum. Electrical force is closer to classical interaction (Coulomb's law), while magnetic force is closer by four orders of magnitude to quantum interaction than electrical one.

To calculate action we should know values of force, distance and time of hydrogen bonds activity. Average val- ues are: force $\approx 2.5 \times 10^{-10} \mathrm{~N}$, distance $\approx 1.6 \times 10^{-10} \mathrm{~m}$ and time $\approx 50 \times 10^{-15} \mathrm{~s}[5,11,12]$. Those values give an action of $h^{*}=F \times d \times t \approx\left(2.5 \times 10^{-10}\right) \times(1.6 \times$ $\left.10^{-10}\right) \times\left(50 \times 10^{-15}\right)=0.5 \times 10^{-33} \mathrm{Js}$, that is a semi-quantum action: Hydrogen bond in water is three orders of magnitude closer to quantum $\left(6.626 \times 10^{-34} \mathrm{Js}\right)$ than to classical $\left(6.626 \times 10^{-30} \mathrm{Js}\right)$ action. According to ratio $F_{\mathrm{M}} / F_{\mathrm{E}} \approx 10^{-4}$, it means that magnetic and electrical fingerprint of hydrogen bond of water will be different, because action of magnetic force will separate it into two pats (quantum and classical), while electrical force will be only classical, because domain of its action is $10^{-29} \mathrm{Js}$ $\left(0.5 \times 10^{-33} \times 10^{4} \approx 10^{-29} \mathrm{Js}\right)$.

To experimentally measure quantum and classical contribution of hydrogen bonds action in the water, we use OMF device (MySkin, USA) and method to separate electrical and magnetic action in light-water interaction [13]. Picture of surface that is taken by classical optical microscope is based on electromagnetic property of light, while OMF is based on difference between diffuse white light (like that of daily light) and reflected polarized light. Reflected polarized light is produced when source of diffuse light irradiates the surface of matter under certain angle (Brewster's angle). Each type of matter has special different angle value of light polarization. We found out that angle of reflected polarized light of water is about $53^{\circ}$ [14]. Since reflected polarized light contains electrical component of light-matter interaction, taking the difference between white light (electromagnetic) and reflected polarized light (electrical) fields gives magnetic properties of matter (opto-magnetic fingerprint).

We used digital images in RGB ( R - red, G - green, $\mathrm{B}$ - blue) system in our analysis, therefore we chose basic pixel data in red and blue channels for white diffuse light $(\mathrm{W})$ and reflected polarized white light $(\mathrm{P})$. Algorithm for data analysis is based on chromaticity diagram called Maxwell's triangle and spectral convolution operation according to ratio of (R-B)\&(W-P). The abbreviated designation means that Red minus Blue wavelength of White light and reflected Polarized light are used in spectral convolution algorithm to calculate data for opto-magnetic fingerprint of matter. Therefore, method and algorithm for creating unique spectral fingerprint are based on the convolution of RGB color channel spectral plots generated from digital images that capture single and multi-wavelength light-matter interaction [13]. To compare magnetic and electrical properties of water (matter) we use to analyze values of Red minus Blue channel for reflected Polarized light, $\mathrm{P}(\mathrm{R}-\mathrm{B})$.

In our experiment we use $18.2 \mathrm{M} \Omega$ water (pure water) with impurities in $p p b$, which gives high percentage of pure hydrogen bond interaction between water molecules.

\section{Experiment and results}

Experiments were done in NanoLab, Faculty of Mechanical Engineering University of Belgrade. Before we start to do experiment we made device calibration and 
checked its reproducibility. We did $24(8 \times 3)$ identical experiments to check parameters value differences. We found that compared to average value the wavelength difference is $\pm 0.14 \mathrm{~nm}$, while the intensity difference is \pm 0.0032 of standardized unit signal.

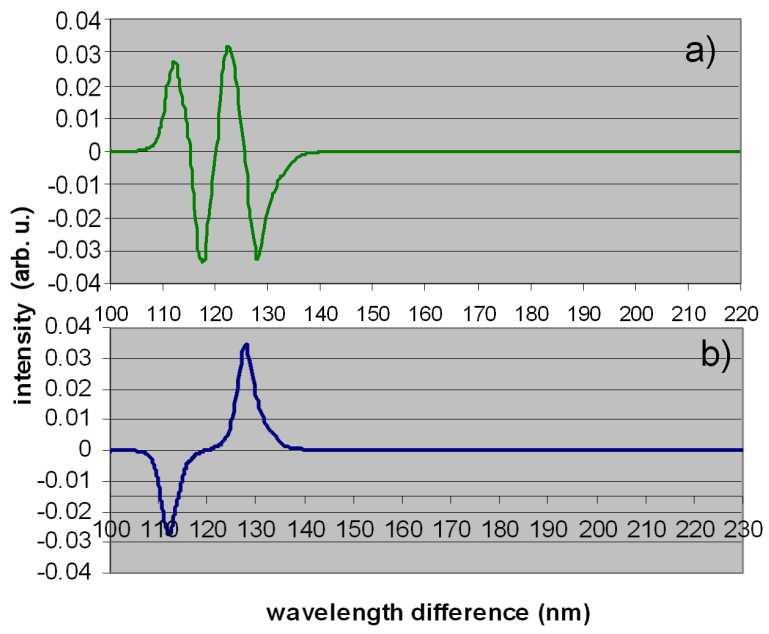

Fig. 1. Opto-magnetic diagrams for $18.2 \mathrm{M} \Omega$ water on $-4.4^{\circ} \mathrm{C}$ : (a) Characteristic points for magnetic domain $[(\mathrm{R}-\mathrm{B}) \&(\mathrm{~W}-\mathrm{P})]$ : $(105.16 \mathrm{~nm}, 0),(111.69 \mathrm{~nm}$, $+0.0256), \quad(114.95 \mathrm{~nm}, 0), \quad(117.07 \mathrm{~nm},-0.0323)$, (120.24 nm, 0), (121.99 nm, 0.0307), (125.49 nm, 0), (127.6 nm, -0.03063), (140.37, 0); (b) Characteristic points for electrical domain $[\mathrm{P}(\mathrm{R}-\mathrm{B})]$ : $(104.01 \mathrm{~nm}, 0)$, $(111.31 \mathrm{~nm},-0.0237),(118.45 \mathrm{~nm}, 0),(127.88 \mathrm{~nm}$, $0.0333),(137.61 \mathrm{~nm}, 0)$

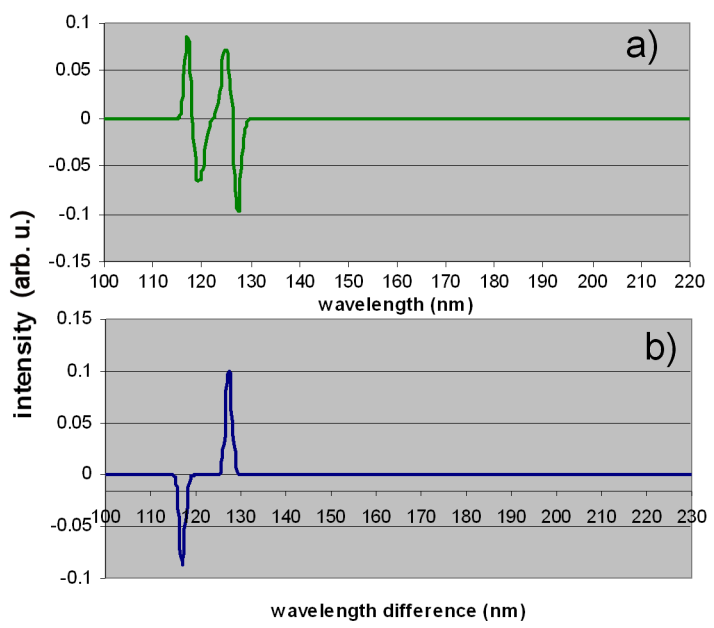

Fig. 2. Opto-magnetic diagram for $18.2 \mathrm{M} \Omega$ water on $25^{\circ} \mathrm{C}$ : (a) Characteristic points for magnetic domain [(R-B)\&(W-P)]: (113.81 nm, 0), (116.69 nm, +0.0781), $(117.95 \mathrm{~nm}, 0),(118.92 \mathrm{~nm},-0.0627),(121.7 \mathrm{~nm}, 0)$, $(124.79 \mathrm{~nm}, \quad 0.0722),(126.19 \mathrm{~nm}, 0), \quad(127.3 \mathrm{~nm}$, $-0.0978),(130.73,0)$; (b) Characteristic points for electrical domain $[\mathrm{P}(\mathrm{R}-\mathrm{B})]$ : $(113.29 \mathrm{~nm}, 0),(116.67 \mathrm{~nm}$, $-0.0782),(118.71 \mathrm{~nm}, 0),(124.16 \mathrm{~nm}, 0),(127.33 \mathrm{~nm}$, 0.1003), (129.07 nm, 0).

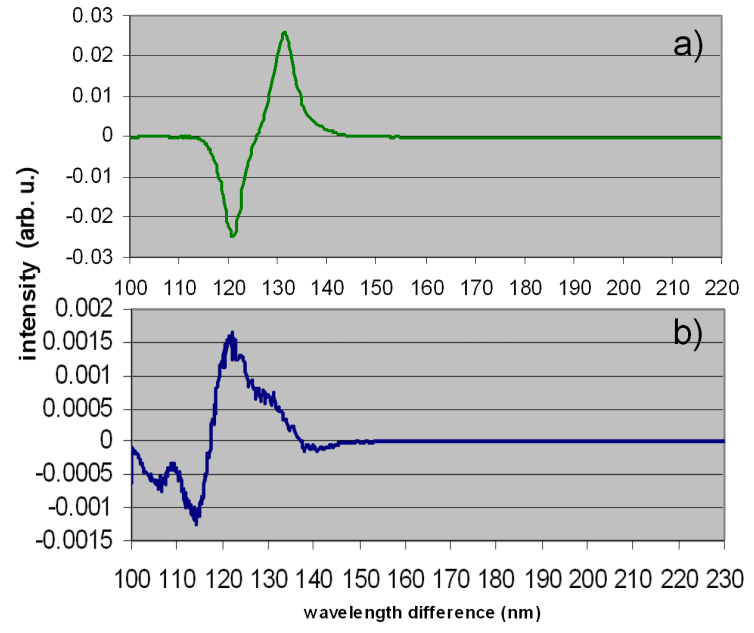

Fig. 3. Opto-magnetic diagram for $18.2 \mathrm{M} \Omega$ water on $50{ }^{\circ} \mathrm{C}$ : (a) Characteristic points for magnetic domain [(R-B)\&(W-P)]: (112.84 nm, 0), (120.49 nm, -0.0241), (125.49 nm, 0), (130.76 nm, 0.0249), (140.76 nm, 0); (b) Characteristic points for electrical domain $[\mathrm{P}(\mathrm{R}-\mathrm{B})]$ : (100.00 nm, 0), (113.42 nm, -0.0011), (116.63 nm, 0), (120.49 nm, 0.0014), (137.61 nm, 0).

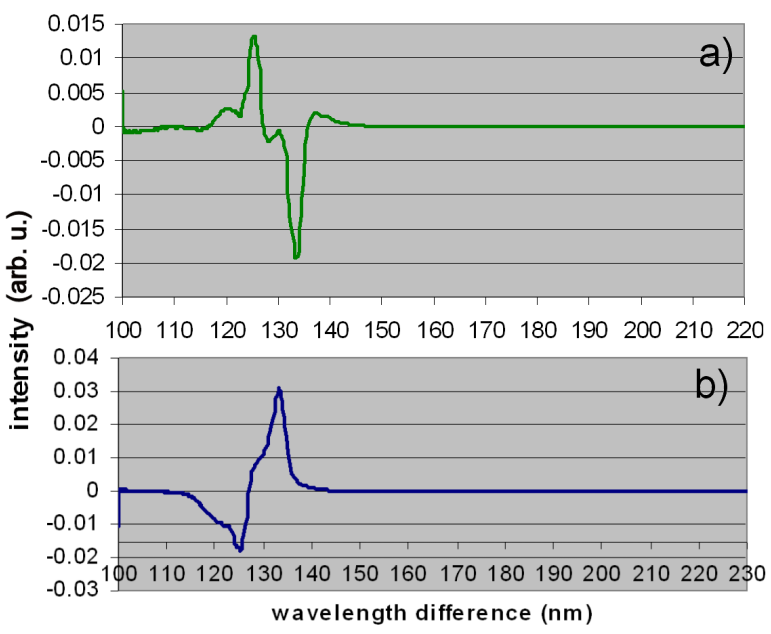

Fig. 4. Opto-magnetic diagram for $18.2 \mathrm{M} \Omega$ water on $50{ }^{\circ} \mathrm{C}$ : (a) Characteristic points for magnetic domain [(R-B)\&(W-P)]: (114.38 nm, 0), (125.26 nm, 0.0131), (127.32 nm, 0), (133.28 nm, -0.0192), (141.51 nm, 0); (b) Characteristic points for electrical domain $[\mathrm{P}(\mathrm{R}-\mathrm{B})]$ : (112.46 nm, 0), (124.16 nm, -0.0149), (126.77 nm, 0), (132.55 nm, 0.0278), (137.61 nm, 0).

In the first experimental step, we characterized $18.2 \mathrm{M} \Omega$ water by $\mathrm{OMF}$ device at different temperatures: $-4.4^{\circ} \mathrm{C}, 25.0^{\circ} \mathrm{C}, 50{ }^{\circ} \mathrm{C}$, and $91.2^{\circ} \mathrm{C}$ (Figs. 1-4). In the second step, we exposed $18.2 \mathrm{M} \Omega$ water at $25^{\circ} \mathrm{C}$ to the influence of constant magnetic field of $50 \mathrm{mT}$ for $9 \mathrm{~min}$ (Fig. 5), and in the third step we exposed waters from the second step to the influence of changeable magnetic field (four times in $9 \mathrm{~min}$ ) with intensity of 40 and $64 \mathrm{mT}$ 


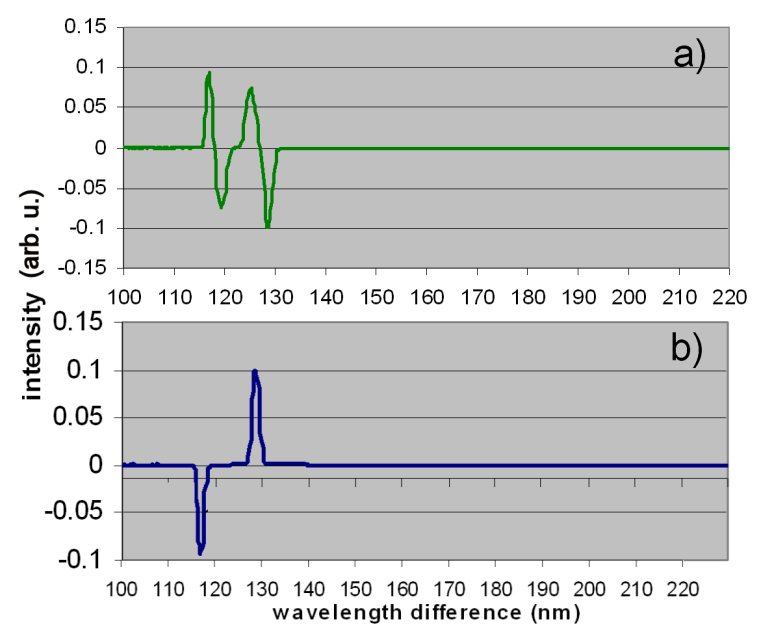

Fig. 5. Opto-magnetic diagram for $18.2 \mathrm{M} \Omega$ water on $25{ }^{\circ} \mathrm{C}$ under influence magnetic feld of $50 \mathrm{mT}$ for 9 min: (a) Characteristic points for magnetic domain [(R-B)\&(W-P)]: (113.80 nm, 0), (116.63 nm, 0.0888), $(117.99 \mathrm{~nm}, 0),(118.96 \mathrm{~nm},-0.0690),(121.22 \mathrm{~nm}, 0)$, $(123.24 \mathrm{~nm}, 0),(124.98 \mathrm{~nm}, 0.0715),(127.10 \mathrm{~nm}, 0)$, $(128.37 \mathrm{~nm},-0.0937),(130.46 \mathrm{~nm}, 0)$; (b) Characteristic points for electrical domain $[\mathrm{P}(\mathrm{R}-\mathrm{B})]$ : $(113.80 \mathrm{~nm}, 0)$, $(116.63 \mathrm{~nm},-0.0889),(118.45 \mathrm{~nm}, 0),(126.32 \mathrm{~nm}, 0)$ (128.37 nm, 0.0939), (130.46 nm, 0).

(Fig. 6). Our results are shown in the system wavelength difference - intensity of the paramagnetic (up peaks) and diamagnetic (down peaks) properties of water. More detailed procedure of the OMF technique is presented in the paper [16], in this issue.

\section{Discussion}

For temperatures $-4.4^{\circ} \mathrm{C}$ and $25^{\circ} \mathrm{C}$ (Figs. 1 and 2) there are two pairs of peaks for magnetic domain, while for electrical there is only one pair (up and down). This suggests that hydrogen bonds possess both classical and quantum properties (sigma bond). For ice this was already observed [3], but we found out that quantum states of hydrogen bond also exist at $25^{\circ} \mathrm{C}$. According to Ref. [3] quantum state of hydrogen bond may have more values of length: $0.172 \mathrm{~nm}, 0.285 \mathrm{~nm}, 0.412 \mathrm{~nm}, 0.510 \mathrm{~nm}$ and $5.80 \mathrm{~nm}$, whose intensities are different. Intensities of the first and second ones are close enough, third one is $15 \%$ of them while forth and fifth ones are $5 \%$ and $3 \%$, respectively. As one can see shape and intensity of electrical interaction are different on $-4.4^{\circ} \mathrm{C}$ and $25^{\circ} \mathrm{C}$ (Figs. $1 \mathrm{~b}$ and $2 \mathrm{~b}$ ).

For temperature $50^{\circ} \mathrm{C}$ sigma bond of hydrogen bond of water molecules on the surface (thin water molecular layer) disappears (suggested by only one pair of peak), because length of hydrogen bonds increases and becomes more than $0.412 \mathrm{~nm}$. However, hydrogen bonds of deeper water molecules keep value $0.412 \mathrm{~nm}$ or less [15]. For hydrogen bond length higher than $0.412 \mathrm{~nm}$ only classical

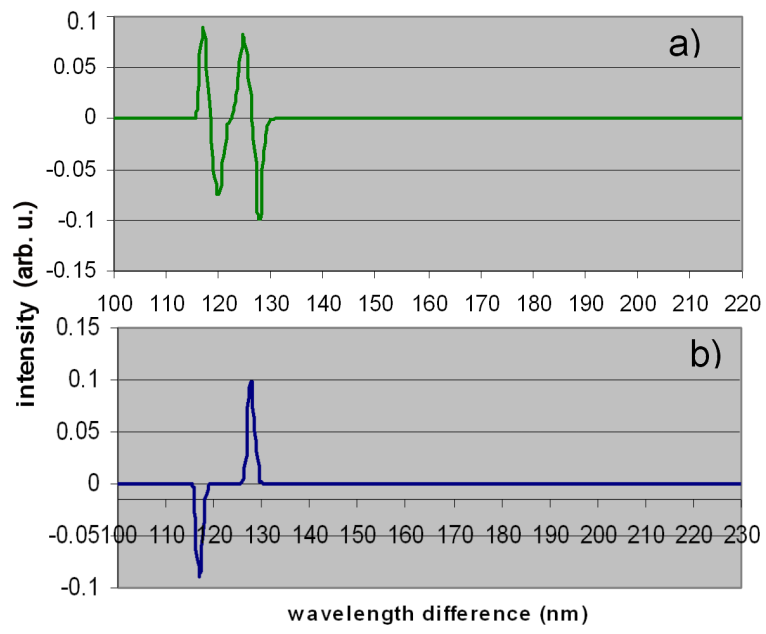

Fig. 6. Opto-magnetic diagram for $18.2 \mathrm{M} \Omega$ water on $25^{\circ} \mathrm{C}$ under influence magnetic feld $40 \mathrm{mT}$ and $64 \mathrm{mT}$ for 9 min: (a) Characteristic points for magnetic domain [(R-B)\&(W-P)]: (114.38 nm, 0), (116.87 nm, 0.0850), $(118.45 \mathrm{~nm}, 0),(119.48 \mathrm{~nm},-0.0702),(121.99 \mathrm{~nm}, 0)$, $(124.43 \mathrm{~nm}, 0.0769),(126.32 \mathrm{~nm}, 0),(127.60 \mathrm{~nm}$, -0.0982), (130.46 nm, 0); (b) Characteristic points for electrical domain $[\mathrm{P}(\mathrm{R}-\mathrm{B})]:(113.60 \mathrm{~nm}, 0),(116.87 \mathrm{~nm}$, $-0.0850),(118.71 \mathrm{~nm}, 0),(125.26 \mathrm{~nm}, 0)(127.60 \mathrm{~nm}$, $0.0987),(130.36 \mathrm{~nm}, 0)$.

interaction exist for both magnetic and electrical interaction (Figs. 3 and 4).

Water becomes sensitive when exposed to the influence of constant magnetic field of $50 \mathrm{mT}$ (Fig. 5), diagram shape for magnetic interaction is a little different and peak values increase for about $15 \%$.

However, when magnetic field changes discretely from 40 to $64 \mathrm{mT}$ (and vice versa) four times in $9 \mathrm{~min}$ (Fig. 6), diagrams of both electrical and magnetic domains become more similar to diagrams when water was without influence of dominant external magnetic field $(50 \mathrm{mT}$ is dominant external magnetic field because Earth magnetic field is about $50 \mu \mathrm{T})$.

Since in general hydrogen bonds possess Golden mean properties [6-8], then investigation of DNA double helix, composed of hydrogen bonds network, has to be goal for future investigations. This would be very important field for medicine (from embryology to steam cell therapy), pharmacy (from better understanding how existing drugs work to design of new drags) and nanotechnology (from materials science to nanomedicine).

\section{Conclusion}

Hydrogen bonds of water molecules until temperature $50^{\circ} \mathrm{C}$ possess both quantum and classical properties, while for higher temperature possess only classical electromagnetic properties.

Under influence of $50 \mathrm{mT}$ (and higher) at $25^{\circ} \mathrm{C}$, hydrogen bonds of water molecules do response. This means 
that water may be treated by magnetic field for its ordering (clustering). Particularly, high interest may be in water clustering and ordering by Golden mean law.

Water structure is important for pharmacy, because of direct implication for drug design. Knowledge of magnetic properties of hydrogen bond, both classical and quantum, may play crucial role for new type drugs design.

All this suggests that more research in hydrogen bonding, molecular recognition, and magnetic and electrical properties of existing drugs is needed.

\section{Acknowledgments}

We acknowledge MySkin (USA) for providing devices to make and analyze digital imaging. This research has been partially funded by Ministry of Science and Technological Development of Republic of Serbia, through Project TR19056.

\section{References}

[1] A. Werner, Liebigs Ann 322, 261 (1902).

[2] L. Pauling, The Nature of the Chemical Bond, Cornel University Press, Ithaca NY 1939.

[3] D. Isaacs, A. Shukla, P. Platyman, D. Hamann, A. Barbiellini, Tulk, Phys. Rev. Lett. 82, 600 (1999).

[4] A. Jeffrey, W. Saenger, Hydrogen Bonding in Biological Structures, Springer-Verlag, Berlin, New York, Heidelberg 1991.
[5] G. Gilli, P. Gilli, The Nature of the Hydrogen Bond: Outline of a Comprehensive Hydrgen Bond Theory, Oxford University Press, Oxford 2009.

[6] D. Yu, D. Xue, H. Ratajczak, J. Mol. Struct. 783, 210 (2006).

[7] R. Heyrovska, Chem. Phys. Lett. 429, 600 (2006).

[8] R. Heyrovska, Chem. Phys. Lett. 436, 287 (2007).

[9] T. Plumridge, R. Waigh, J. Pharmacy $\&$ Pharmacology 54, 1155 (2002).

[10] Dj. Koruga, A. Tomic, Z. Ratkaj, L. Matija, Mat. Sci. Forum 518, 491 (2006).

[11] S. Chang, J. He, A. Kibel, M Lee, O. Sankey, P. Yhang, S. Lindsay, Nature Nanotechnology 4, 297 (2009).

[12] M. Cowan, B. Bruner, N. Huse, J. Dwyer, B. Chugh, E. Nibbering, T. Elsaesser, R. Miller, Nature 434, 199 (2005).

[13] Dj. Koruga, A. Tomic, Method and algorithm for analysis of light-matter interaction based on spectral convolution, US Pat. App. No. 61/061, 852, 2008, PCT/ US2009/030347, Publ. No: WO/2009/089292, Publ. Date: 2009-07-16.

[14] D. Lide, Handbook of Chemistry and Physics, 77th Edition, CRC Press, Boca Raton 1996.

[15] G. Hura, D. Russo, R.M. Glaeser, T. Head-Gordon, M. Krack, M. Parrinello, Phys. Chem. Chem. Phys. 5, 1981 (2003).

[16] M. Papić-Obradović, D. Kojić, L. Matija, Acta Phys. Pol. A 117, 782 (2010). 